\title{
Multiple interactions, diffraction, and the BFKL pomeron
}

\author{
Gösta Gustafson \\ Dept. of Theoretical Physics, Lund University, Lund, Sweden \\ E-mail: gosta.gustafson@thep.lu.se
}

DOI: http://dx.doi.org/10.5689/UA-PROC-2010-09/33

\begin{abstract}
In high energy $p p$ scattering the inclusive cross section for minijet production is very large. Unitarity then implies a high probability for multiple interactions, and a large diffractive cross section. As the proton has a substructure in terms of parton cascades, the diffraction is not only elastic, but has a large component of diffractive excitation. The cascades can fill the whole rapidity range between projectile and target, and in the Good-Walker formalism the diffractive excitation is determined by the fluctuations in the evolution. Within the Lund Dipole Cascade model, it is in this way possible to reproduce diffractive excitation in $p p$ collisions and DIS. The result shows a multi-regge behavior, with a bare pomeron pole with $\alpha(0)=1.21, \alpha^{\prime}=0.2 \mathrm{GeV}^{-2}$, and an almost constant triple-pomeron coupling $g_{3 P} \approx 0.3 \mathrm{GeV}^{-1}$. It is also pointed out that the classification of diffractive events varies between different formalisms, and cannot be uniquely defined. Therefore it is recommended to study gap events rather than diffractive events. The results presented in this talk are obtained in collaboration with Christoffer Flensburg and Leif Lönnblad.
\end{abstract}

\section{Introduction}

It is often assumed that high energy collisions are driven by partonic subcollisions. This is e.g. the case in the model implemented in the frequently used MC event generator PYTHIA [1]. In high energy $p p$ scattering the cross section for minijet production becomes very large, and unitarity implies that multiple interactions, saturation, and diffraction become important. Effects of saturation and multiple interactions are most easily described in impact parameter space, as parton rescattering is represented by a convolution in transverse momentum space, which corresponds to a simple multiplication in transverse coordinate space.

The proton has an internal substructure, which may be excited in a diffractive scattering process, and diffractive excitation represents large fractions of the cross section in $p p$ collisions or DIS. In the Good-Walker formalism [2] diffractive excitation is described by the fluctuations in the scattering amplitude. In most analyses of $p p$ collisions this mechanism is used only for low mass excitation, while high mass excitation is described by a triple-regge formula [3, 4], where regge trajectories and couplings are fitted to experimental data (for recent analyses see e.g. Refs. $[5,6,7])$.

The proton substructure is represented by a parton cascade, which at high energies is described by BFKL evolution. The fluctuations in this evolution are known to be very large [8]. An analysis of these fluctuations, within the Lund Dipole Cascade model, is able to reproduce 
the experimental cross sections for diffractive excitation in $p p$ collisions or DIS [9, 10]. This implies that the effective pomeron couplings in the multi-pomeron formalism can be estimated without any new free parameters.

It should also be noticed that the classification of diffractive events varies between different formalisms, and cannot be uniquely defined. Therefore it is recommended to study gap events rather than diffractive events.

\section{The eikonal approximation and the Good-Walker for- malism}

As mentioned in the introduction, diffraction, saturation, and multiple interactions are most easily described in impact parameter space. If the scattering is driven by absorption into inelastic states $i$, with weights $2 f_{i}$, the elastic amplitude is given by

$$
T=1-e^{-F}, \text { with } F=\sum f_{i} .
$$

For a structureless projectile we find:

$$
\left\{\begin{array}{l}
d \sigma_{\text {tot }} / d^{2} b=\langle 2 T\rangle \\
\sigma_{\text {el }} / d^{2} b=\langle T\rangle^{2} \\
\sigma_{\text {inel }} / d^{2} b=\left\langle 1-e^{-\sum 2 f_{i}}\right\rangle \sim \sigma_{t o t}-\sigma_{e l} .
\end{array}\right.
$$

If the projectile has an internal structure, the mass eigenstates $\Psi_{k}$ can differ from the eigenstates of diffraction $\Phi_{n}$, which have eigenvalues $T_{n}$. With the notation $\Psi_{k}=\sum_{n} c_{k n} \Phi_{n}$ (with $\Psi_{i n}=\Psi_{1}$ ) the elastic amplitude is given by $\left\langle\Psi_{1}|T| \Psi_{1}\right\rangle=\sum c_{1 n}^{2} T_{n}=\langle T\rangle$, while the amplitude for diffractive transition to mass eigenstate $\Psi_{k}$ is given by $\left\langle\Psi_{k}|T| \Psi_{1}\right\rangle=\sum_{n} c_{k n} T_{n} c_{1 n}$. The corresponding cross sections become

$$
\begin{aligned}
d \sigma_{e l} / d^{2} b & =\left(\sum c_{1 n}^{2} T_{n}\right)^{2}=\langle T\rangle^{2} \\
d \sigma_{d i f f} / d^{2} b & =\sum_{k}\left\langle\Psi_{1}|T| \Psi_{k}\right\rangle\left\langle\Psi_{k}|T| \Psi_{1}\right\rangle=\left\langle T^{2}\right\rangle .
\end{aligned}
$$

The diffractive cross section here includes elastic scattering. Subtracting this gives the cross section for diffractive excitation, which is determined by the fluctuations in the scattering process:

$$
d \sigma_{d i f f e x} / d^{2} b=d \sigma_{d i f f}-d \sigma_{e l}=\left\langle T^{2}\right\rangle-\langle T\rangle^{2} .
$$

\section{Dipole cascade models}

Mueller's dipole cascade model $[11,12,13]$ is a formulation of BFKL evolution in transverse coordinate space. Gluon radiation from the color charge in a parent quark or gluon is screened by the accompanying anticharge in the color dipole. This suppresses emissions at large transverse separation, which corresponds to the suppression of small $k_{\perp}$ in BFKL. For a dipole $(\mathbf{x}, \mathbf{y})$ the probability per unit rapidity $(Y)$ for emission of a gluon at transverse position $\mathbf{z}$ is given by

$$
\frac{d \mathcal{P}}{d Y}=\frac{\bar{\alpha}}{2 \pi} d^{2} \mathbf{z} \frac{(\mathbf{x}-\mathbf{y})^{2}}{(\mathbf{x}-\mathbf{z})^{2}(\mathbf{z}-\mathbf{y})^{2}}, \quad \text { with } \bar{\alpha}=\frac{3 \alpha_{s}}{\pi} .
$$




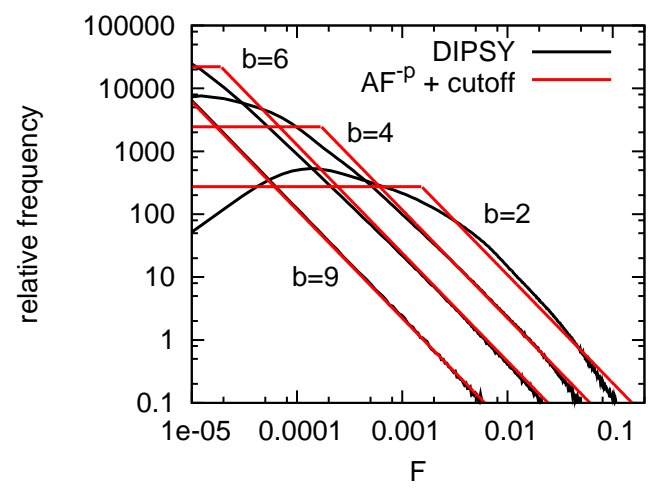

Figure 1: Distribution in the one-pomeron amplitude $F$ in DIS for $Q^{2}=14 \mathrm{GeV}^{2}$ and $W=220$ $\mathrm{GeV}$. The photon is here represented by a dipole with size $r=1 / Q$. The impact parameter $b$ is measured in $\mathrm{GeV}^{-2}$.

The dipole is split into two dipoles, which (in the large $N_{c}$ limit) emit new gluons independently. The result is a cascade, where the number of dipoles grows exponentially with $Y$.

When two cascades collide, a pair of dipoles with coordinates $\left(\mathbf{x}_{i}, \mathbf{y}_{i}\right)$ and $\left(\mathbf{x}_{j}, \mathbf{y}_{j}\right)$ can interact via gluon exchange with the probability $2 f_{i j}$, where

$$
f_{i j}=f\left(\mathbf{x}_{i}, \mathbf{y}_{i} \mid \mathbf{x}_{j}, \mathbf{y}_{j}\right)=\frac{\alpha_{s}^{2}}{8}\left[\log \left(\frac{\left(\mathbf{x}_{i}-\mathbf{y}_{j}\right)^{2}\left(\mathbf{y}_{i}-\mathbf{x}_{j}\right)^{2}}{\left(\mathbf{x}_{i}-\mathbf{x}_{j}\right)^{2}\left(\mathbf{y}_{i}-\mathbf{y}_{j}\right)^{2}}\right)\right]^{2} .
$$

Summing over all dipoles in the cascades then reproduces the LL BFKL result. The elastic scattering amplitude is given by $T=1-\exp \left(-\sum f_{i j}\right)$, and the cross sections are given by Eqs. $(2,3,4)$.

The Lund cascade model $[14,15,16]$ is a generalization of Mueller's model, which includes:

- NLL BFKL effects

- Nonlinear effects in the evolution

- Confinement effects

For an incoming virtual photon splitting in a $q \bar{q}$ pair, the initial state wavefunction is determined by perturbative QCD. For an incoming proton we make an ansatz in form of an equilateral triangle of dipoles. After evolution the result is rather insensitive to the exact form of the initial state. The model is also implemented in a MC program DIPSY. The model reproduces successfully the total and (quasi)elastic cross sections for DIS and $p p$ scattering.

\section{Fluctuations and diffractive excitation}

The fluctuations in the evolution are large, and the model can also describe diffractive excitation within the Good-Walker formalism, without new parameters beyond those adjusted to the total and elastic cross sections [9]. This is similar in spirit to the early analysis by Miettinen and Pumplin [17]. In DIS saturation effects are not very important, while in $p p$ collisions saturation effects strongly suppress the fluctuations, and thus the cross section for diffractive excitation. 

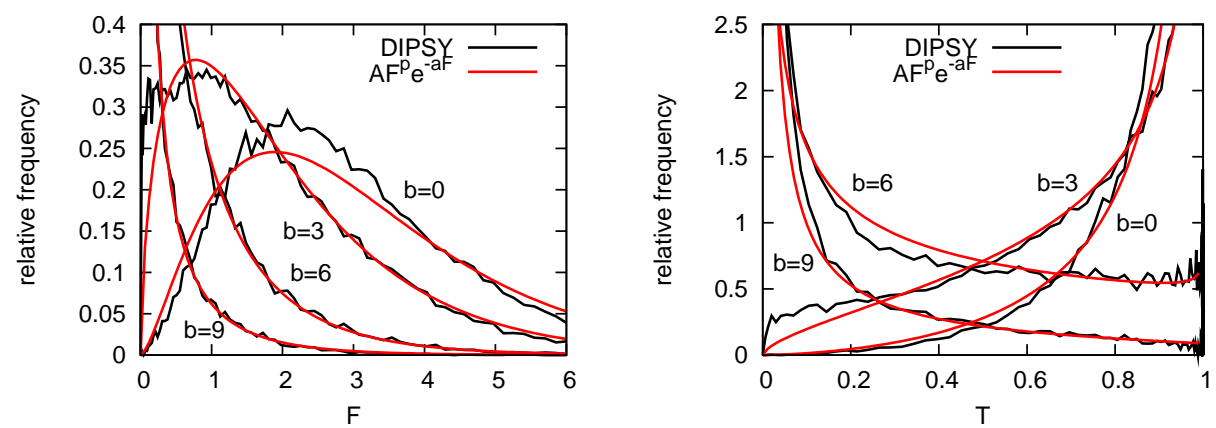

Figure 2: Distribution in the one-pomeron amplitude ( $F$, left) and the uniterized amplitude $(T$, right) in $p p$ collisions at $2 \mathrm{TeV}$. Notation as in Fig. 1.

\section{$\gamma^{*} p$ collisions}

The distribution in the non-saturated scattering amplitude, $F$, is shown in Fig. 1 for different impact parameters. The distribution can be approximately described by a power $\frac{d P}{d F} \approx A F^{-P}$ (with a cutoff for small $F$-values), which is illustrated by the straight lines in the figure. The width of this distribution is rather large, and the approximation gives the ratio $d \sigma_{\text {diff.ex. }} / d \sigma_{t o t} \approx 1-1 / 2^{2-p}$. In the simulations the power $p$ is rather independent of the impact parameter, and therefore this result is also valid for the integrated cross sections. This gives $\sigma_{\text {diff }} / \sigma_{\text {tot }} \sim 0.13$ at $Q^{2}=50 \mathrm{GeV}^{2}$, decreasing for larger $Q^{2}$, but fairly insensitive to the energy $W$.

\section{$p p$ collisions}

In $p p$ scattering the Born amplitude is large, and therefore unitarity effects are important. Figure 2 shows both the Born amplitude and the unitarized amplitude at $2 \mathrm{TeV}$ for different $b$-values. We see that the width of the Born amplitude is large, and without unitarization the fraction of diffractive excitation would be similar to that for $\gamma^{*} p$ for lower $Q^{2}$-values. (The smooth lines are fits of the form $A F^{p} e^{-a F}$.)

However, the unitarized amplitude is limited by 1 , and the width, and therefore the diffractive excitation, is very much reduced. This is in particular the case for central collisions where the amplitude approaches the black disc limit, as seen in the right panel in Fig. 2. This result corresponds to the effect of enhanced diagrams in the conventional triple-regge approach. The impact parameter profile is shown in Fig. 3. We see that the cross section for diffractive excitation is largest in a ring with radius $b \sim 1 \mathrm{fm}$. This result also implies that factorization is not satisfied when comparing diffractive excitation in DIS and $p p$ scattering.

\section{Comparison with multi-regge analyses}

It is also interesting to compare the results from the Good-Walker analysis with the multi-regge formalism. To this end we study the contribution from the bare pomeron, meaning the onepomeron amplitude without contributions from saturation, enhanced diagrams or gap survival form factors. When $s, M_{\mathrm{X}}^{2}$, and $s / M_{\mathrm{X}}^{2}$ are all large, pomeron exchange should dominate. The results of the $\mathrm{MC}$ for the total, elastic, and single diffractive cross sections are shown by the crosses in the right panel in Fig. 3. We note that the results are very well reproduced by a 

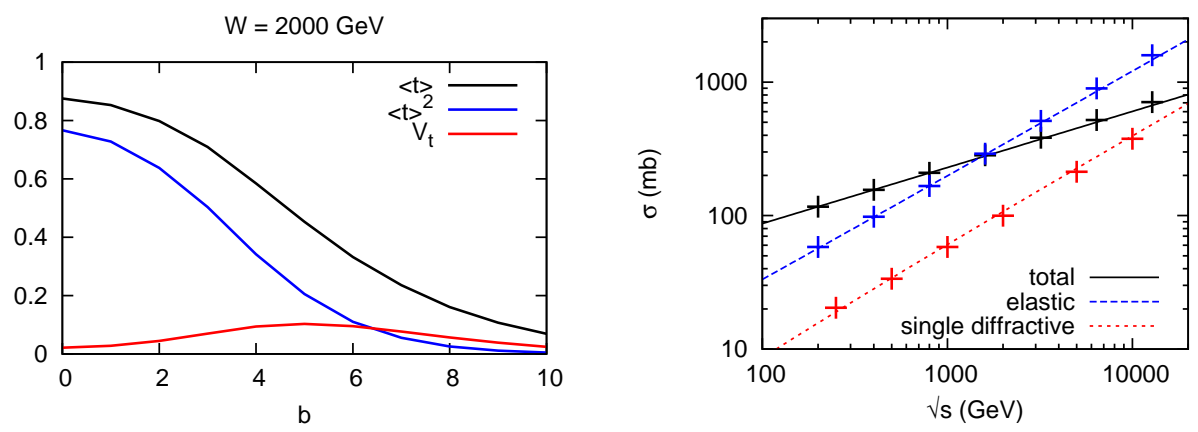

Figure 3: Left: Impact parameter distributions for $\langle T\rangle=\left(d \sigma_{\mathrm{tot}} / d^{2} b\right) / 2,\langle T\rangle^{2}=d \sigma_{\mathrm{el}} / d^{2} b$, and $V_{T}=d \sigma_{\text {diffex }} / d^{2} b$ in $p p$ collisions at $W=2 \mathrm{TeV} . b$ is in units of $\mathrm{GeV}^{-1}$. Right: The total, elastic, and single diffractive cross sections in the one-pomeron approximation. The crosses are model calculations and the lines are from a tuned triple-regge parametrisation.

triple-regge expression with a single pomeron pole, with the parameters

$$
\alpha(0)=1.21, \quad \alpha^{\prime}=0.2 \mathrm{GeV}^{-2}, \quad g_{\mathrm{pP}}(t)=\left(5.6 \mathrm{GeV}^{-1}\right) e^{1.9 t}, \quad g_{3 \mathrm{P}}(t)=0.31 \mathrm{GeV}^{-1},
$$

which is shown by the straight lines. Also the $t$-dependence of diffractive excitation is well reproduced.

These results can also be compared with multi-regge analyses, where e.g. Ryskin et al. [5] obtain $\alpha(0)=1.3, \alpha^{\prime} \leq 0.05 \mathrm{GeV}^{-2}$, Kaidalov et al. [6] find $\alpha(0)=1.12, \alpha^{\prime}=0.22 \mathrm{GeV}^{-2}$, while Gotsman et al. [7] find $\alpha(0)=1.335, \alpha^{\prime}=0.01 \mathrm{GeV}^{-2}$. Thus our values are somewhere in between. We also note that the Good-Walker results are reproduced by a single pomeron pole, i.e. not by a cut as expected in LL BFKL, or a series of poles as obtained with a running coupling [18]. Also the triple-regge coupling $g_{3 \mathrm{P}}$ is approximately constant, while in LL BFKL it is proportional to $\sim 1 / \sqrt{|t|}[12,19]$.

\section{Can diffraction be uniquely defined?}

Multipomeron diagrams are included in the dipole picture, with fixed multi-pomeron couplings. However, all events with no gap are classified as inelastic. This can be compared to the formalism in Ref. [5], in which a large cross section for overlapping double diffraction is obtained.

To compare predictions from different models with each other and with experiments, we need a unique definition of diffraction. One attempt might be events with two separate color singlet systems, containing the original valence quarks. This could be obtained by exchange of two (or more) gluons forming a color singlet. If such a state is obtained in a calculation in perturbative QCD, a gap could, however, be filled by final state radiation or nonperturbative strings, or else a gap could be formed by color reconnection. Thus diffractive events cannot be uniquely defined by perturbative QCD. The definition varies between different schemes, and for a specified event the diffractive capacity is not an observable. The solution must be: study observables, meaning events with a gap. 


\section{Summary}

- In high energy $p p$ scattering unitarity implies a high probability for multiple interactions, and a large diffractive cross section. In the Good-Walker formalism elastic scattering is given by the average of the scattering amplitude, while the fluctuations determine the diffractive excitation.

- Parton cascades fill the whole rapidity range between projectile and target. The fluctuations in BFKL evolution are large, and within the Lund Dipole Cascade model they can describe diffractive excitation within the Good-Walker formalism, to both low and high masses.

- When the interaction approaches the black disc limit in central $p p$ collisions, fluctuations and diffractive excitation is suppressed. This leads to factorization breaking in comparisons of DIS and $p p$ scattering.

- The result of the model calculations corresponds to a bare pomeron, which is a simple pole, and an almost constant triple-pomeron coupling.

- Diffractive excitation is scheme dependent, and cannot be uniquely defined. Study gap events.

\section{References}

[1] T. Sjostrand and M. van Zijl, Phys. Rev. D36 (1987) 2019.

[2] M.L. Good and W.D. Walker, Phys. Rev. 120 (1960) 1857.

[3] A.H. Mueller, Phys. Rev. D2 (1970) 2963.

[4] C.E. DeTar et al., Phys. Rev. Lett. 26 (1971) 675.

[5] M.G. Ryskin, A.D. Martin, and V.A. Khoze, Eur. Phys. J. C60 (2009) 249 [arXiv:0812.2407]

[6] A.B. Kaidalov and M.G. Poghosyan, arXiv:0909.5156.

[7] E. Gotsman, E. Levin, U.Maor, and J.S.Miller Eur. Phys. J. C57 (2008) 689 [arXiv:0805.2799].

[8] A.H. Mueller and G.P. Salam, Nucl. Phys. B475 (1996) 293 [hep-ph/9605302].

[9] E. Avsar, G. Gustafson, and L. Lönnblad, JHEP 12 (2007) 012 [arXiv:0709.1368].

[10] C. Flensburg and G. Gustafson, JHEP 1010 (2010) 014 [arXiv:1004.5502].

[11] A.H. Mueller, Nucl. Phys. B415 (1994) 373.

[12] A.H. Mueller and B. Patel, Nucl. Phys. B425 (1994) 471 [hep-ph/9403256].

[13] A.H. Mueller, Nucl. Phys. B437 (1995) 107 [hep-ph/9408245].

[14] E. Avsar, G. Gustafson, and L. Lönnblad, JHEP 07 (2005) 062 [hep-ph/0503181].

[15] E. Avsar, G. Gustafson, and L. Lönnblad, JHEP 01 (2007) 012 [hep-ph/0610157].

[16] C. Flensburg, G. Gustafson, and L. Lönnblad, Eur. Phys. J. C60 (2009) 233, [arXiv:0807.0325].

[17] H.I. Miettinen and J. Pumplin, Phys. Rev. D18 (1978) 1696.

[18] L.N. Lipatov, Sov. Phys. JETP 63 (1986) 904.

[19] J. Bartels, M.G. Ryskin, and G.P. Vacca, Eur. Phys. J. C27 (2003) 101, [hep-ph/0207173] 